This item was submitted to Loughborough's Institutional Repository (https://dspace.lboro.ac.uk/) by the author and is made available under the following Creative Commons Licence conditions.

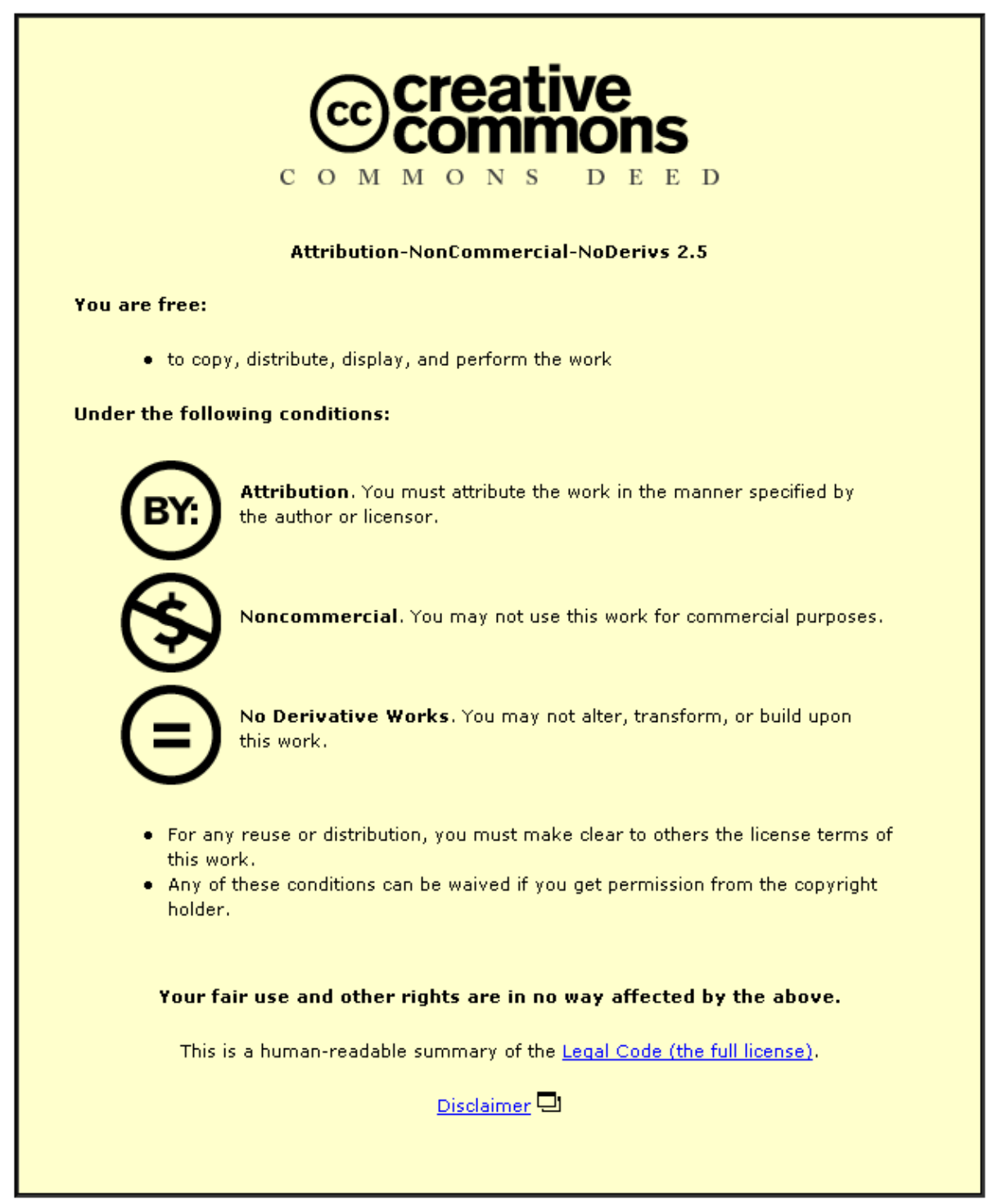

For the full text of this licence, please go to: http://creativecommons.org/licenses/by-nc-nd/2.5/ 


\title{
The Energy Absorbed in the Human Head Due to Ring-Type Jewelry and Face-Illuminating Mobile Phones Using a Dipole and a Realistic Source
}

\author{
William G. Whittow, Chinthana J. Panagamuwa, Robert M. Edwards, Senior Member, IEEE, and \\ John (Yiannis) C. Vardaxoglou
}

\begin{abstract}
The effect of facial ring jewelry is investigated on the energy absorbed in the head when illuminated by communicationsenabled personal data assistant (PDA)-type devices. We study the relative changes in specific absorption rates in the head due to perturbing metallic rings in proximity to the face illuminated by a $1.8 \mathrm{GHz}$ dipole and a monopole on a conducting box. Simple and complex head models used in a validated finite-difference time-domain (FDTD) simulation are compared with measurements from an industry standard DASY4 SAR measurement system. Both simulation and measurement are referred to the IEEE specific anthropomorphic mannequin head. Results show that metallic rings may alter specific absorption rates (SAR) level distributions within the head. Results are given for several common ring sizes.
\end{abstract}

Index Terms-DASY4, finite-difference time-domain (FDTD), metallic jewelry, personal data assistant (PDA), specific absorption rates $(\mathbf{S A R})$.

\section{INTRODUCTION}

$\mathbf{T}$ HE study of interactions between biological material and the energy generated by personal communications devices is currently topical. More recently, personal data assistant (PDA)-type devices are becoming available that are designed to be held in front of the face rather than close to the ear. By observation, it can be seen that jewelry is popular and that the closed ring, sometimes referred to as the magnetic dipole, is one of the most common forms of jewelry. It has been known for some years, that a proportion of the radio frequency energy from mobile phones is absorbed in the head and several papers have discussed distributions of specific absorption rates (SAR) in the head. In this paper, it will be shown that rings can redistribute the absorbed energy and focus it into smaller regions of biological matter.

This paper investigates the field perturbation effects of mobile communication enabled devices (MCED), for example, PDAs, due to thin wire jewelry rings of various common diameters. These type of devices are different from normal phones in that they are designed to be used in front of the face rather than close

Manuscript received October 19, 2007; revised April 28, 2008. Current version published December 30, 2008.

The authors are with the Department of Electronic and Electrical Engineering Department, Loughborough University, Leicestershire LE11 3TU, U.K. (e-mail: r.m.edwards@lboro.ac.uk).

Color versions of one or more of the figures in this paper are available online at http://ieeexplore.ieee.org.

Digital Object Identifier 10.1109/TAP.2008.2007353 to the ear. A continuous wave $(\mathrm{CW})$ source is used to simulate a communications-enabled PDA positioned in front of the head; (possible effects of a wire or a Bluetooth link to an earpiece have not been considered in this paper). Considered discussion is given to the choice of numerical electromagnetic phantom models and the choice of representative sources. Intuitively, it would seem that for this type of research a realistic source would produce realistic results. However, the range of sources is so diverse that such a source has not yet been agreed upon. We have, therefore, chosen to refer all of our results to the agreed standard of the IEEE specific anthropomorphic mannequin (SAM) head. This allows for simple comparison and simple measurement of the likely main effects of ring type jewelry on the SAR in the head.

Typically, mobile phones research has been conducted for MCED positioned near the ear [1]-[4]. However, the head has also been irradiated from in front of the eye using realistic mobile phone models [3]-[5]. The authors of this paper have also briefly considered the effect of metallic jewelry on the SAR in the head [6], [7] and this paper greatly extends that work. Metallic ear rings have previously been considered with a source by the ear [8]-[10].

Metallic implants inside the head have been found to increase the SAR [11], [12]. Virtanen considered metallic loops and pins inside a cylindrical head [12]. The same author has reviewed the area of passive implants [13] and investigated implants in a heterogeneous anatomical model of a head [8]. Note, results for implants are not included in this paper.

In relation to sources, the radiation efficiency of a dipole can be changed by using a passive reflector and directive element [14]. Tay found that the efficiency of the reflector could be increased if a metallic scatterer was positioned near to a dipole. The head has also been irradiated with dipoles positioned near metallic walls with a geometric head [15] and an anatomical head [16]. Both papers showed that metallic walls could increase the power absorbed in the head.

The authors have previously considered the effects on SAR of metallic spectacles with a source positioned in front of the face [17], [18]. The finite-difference time-domain (FDTD) method has been used to examine thin metallic spectacles on a heterogeneous phantom [19], [20]. The excitation used was a monopole on a metallic box positioned at the side of the head. These papers showed that metallic spectacles may redistribute energy produced by an MCED. Wang [20] postulated that increased SAR in parts of the head were due to current on the spectacles. The 


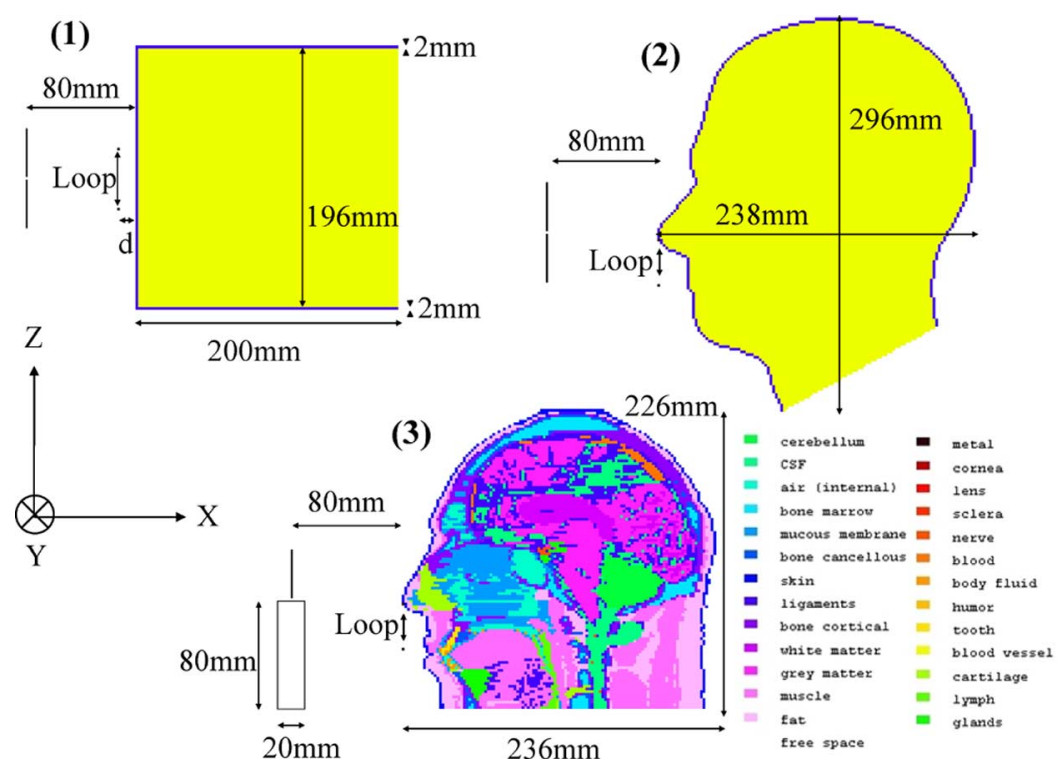

Fig. 1. Orientation and geometry of source, ring, and head.

FDTD method with scaled models of an adult head was used to consider the effects of metallic spectacles on adults and children with a mobile phone placed by the ear [21].

Measurements with a phantom and metallic spectacles showed that spectacles can affect the level of radiation near the eyes by $\pm 20 \mathrm{~dB}$ due to shielding, enhancement, and depolarization effects [22]. For MCED operating at $835 \mathrm{MHz}$ held by the ear, the SAR measured in the eye closest to the phone was found to increase by almost $30 \%$ [23].

\section{A. Structure of This Paper}

This paper is structured as follows. Section II-A contains a description of the type of sources used for our experiments. In Section II-B, the choice of simulation phantoms is discussed where the types and properties of models in FDTD are mentioned.

The first simulated results are presented in Section III-A for an homogenous cubic head with jewelry rings. It is in this abstract system that the focusing effects of rings on SAR are most easily seen. In Section III-B, measurements are presented for rings with an homogeneous SAM head. In the same section, measurements with the SAM head are compared with simulation results and agreement is obtained. Then in Section III-C, simulation results are presented for a more anatomically accurate phantom head and a more realistic source. Finally, in Section IV, conclusions of the results are discussed.

\section{DESCRIPTION OF SimUlation}

Description of the FDTD code used in this paper can be found in [17], [18]. Perfectly matched layers (PML) with geometric grading [24] are used as absorbing boundary conditions to terminate the grid. The PML is eight cells thick and is positioned at least 12 cells from the head. The Yee cell size used throughout this paper is $2 \mathrm{~mm}$. The lowest number of cells per wavelength was always greater than 10 , and reasonable results have been obtained with only four [25]. The time step was $3.336 \mathrm{pS}$. The simulations were run for at least 10 cycles and until stability was achieved (at least 1660 time steps). In FDTD, the metallic jewelry rings were modeled using Yee cells with the conductivity of copper [17].

\section{A. Dipole and Monopole Source Simulation}

Two different sources have been used in this paper; a dipole and a monopole on a metallic box.

Papers in this area of research have tended to use sources that fall into two distinct groups. Sources that have associated phone structures for example a metal box and perhaps some form of modulation; and more generic sources such as plane waves, monopoles and dipoles. $\lambda / 2$ dipole models at $1.8 \mathrm{GHz}$ have been used initially as the excitation source in this paper. Previous researchers using the same excitation include [26], [27]. The use of a dipole source allows the simulated results to be compared with measurements. Here, in both measurement and simulation, a dipole has been positioned several centimeters away from the head and the jewelry. Therefore, the choice of excitation is less significant than in simulations where the phone is positioned next to the head, as the head will have less effect on the currents on the antenna and casing. Since the dipole is well understood, a dipole source allows the differences in absorption to be attributed properly to the jewelry rather than the source and its associated realistic metal box. The dipoles used here are vertically orientated along the $\mathrm{Z}$ axis, see Fig. 1. Note, Section III-B also uses a horizontally orientated dipole (along the $\mathrm{Y}$ axis).

In Section III-C, a 1/4 wave monopole on a box was used to replicate a realistic phone when rings were added to the anatomically realistic head, see Fig. 1. Tinniswood showed that a realistic phone can be accurately represented by a monopole on a metal box covered in plastic [1]. As the source in this paper was positioned several centimeters from the head, the plastic cover is less important and was not included. The monopole was positioned at the center of the top of the perfectly electrically conducting box. The dimensions of the box were $20 x \times 40 y \times$ $80 z(\mathrm{~mm})$. This same monopole source with slightly different sizes of metal box has often been used in the literature [21], [28], 
[29]. A model of the hand has not been included. This reduces the computational complexity and runtime [3].

The dipoles and monopoles are fed at their center with a sinusoidal CW source and all results are normalized to $1 \mathrm{~W}$. Note that there are a number of duty factors associated with mobile phones which concludes to the scaling of the results by an appropriate fraction [3], [4]. $1 \mathrm{~W}$ radiated power is commonly used as it allows the results to be easily scaled as the SAR results are already in units of $\mathrm{W} / \mathrm{kg}$ per $\mathrm{W}[3]$.

A study using twenty adult volunteers was undertaken to measure the typical distance between the eye and screen of a PDA device when a user was reading text. The worst case scenario with the PDA closest to the eyes was $100 \mathrm{~mm}$. The dipole, $100 \mathrm{~mm}$ from the eyes was $80 \mathrm{~mm}$ in front of the tip of the nose. Therefore, the source was placed at a distance of $80 \mathrm{~mm}$. This was considered to be a reasonable experimental method and was usefully representative. Using the condition $k r \cong 1$ for the near field boundary in which $k$ is the wavenumber and $r$ is the distance between field point and source; we see that our jewelry lies in the Fresnel region at $1.8 \mathrm{GHz}$.

\section{B. The Head Models}

In this paper, three different types of head models have been used. The cubical phantom was a $200 \mathrm{~mm}$ cubic homogenous head with the properties of brain simulating tissue $\left(\sigma=1.37 \mathrm{~S} / \mathrm{m}, \varepsilon \mathrm{r}=40.48, \rho=1000 \mathrm{~kg} / \mathrm{m}^{3}\right)$ [IEEE 1528] with a $2 \mathrm{~mm}$ outer shell made of fiberglass $(\sigma=0 \mathrm{~S} / \mathrm{m}$, $\left.\varepsilon_{\mathrm{r}}=3.5\right)$. This Phantom allowed us to investigate rings which were a constant distance from the head and therefore, reduced the complexity of the coupling mechanism.

Phantom 2 was a SAM Phantom defined in IEEE 1528-2003, CENELEC 50361, and IEC 62209. For this phantom, we have used both a computational model for FDTD simulation and a physical model for measurement. This phantom allowed our simulation results to be compared with measurements using a modified rear-entry SAM head developed and tested by the authors [30], [31].

Phantom 3 is an anatomically realistic head provided by Brooks Air Force (www.brooks.af.mil/). The 3-D head, which is based on The Visible Human Project, is that of an adult male and has 25 tissue types. The tissue properties at $1.8 \mathrm{GHz}$ are given in [18]. The head data has a $2 \mathrm{~mm}$ resolution. The three geometries used in this paper are shown in Fig. 1 and are as follows:

1) a $74 \mathrm{~mm}$ dipole $80 \mathrm{~mm}$ in front of a $200 \mathrm{~mm}$ homogeneous cubical phantom with a $2 \mathrm{~mm}$ fiberglass shell;

2) a $74 \mathrm{~mm}$ dipole $80 \mathrm{~mm}$ in front of the tip of the nose of the SAM head; and

3) a 1/4 wave monopole (the length of the monopole was $38 \mathrm{~mm}$ including a $2 \mathrm{~mm}$ gap across which a sinusoidal source was used), on a metallic box $80 \mathrm{~mm}$ in front of the tip of the nose of an anatomically realistic head.

\section{RESULTS}

\section{A. Varying Ring Size and Distance to Cubical Phantom $(1.8 \mathrm{GHz})$}

In this experiment, the cubical phantom in Fig. 1 was used. With a fixed vertically aligned dipole, the peak 1-g SAR was

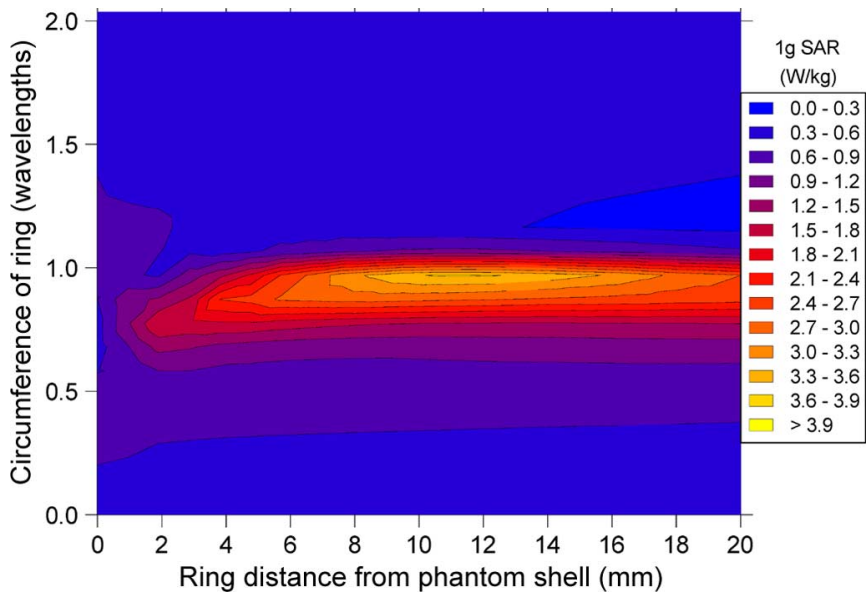

Fig. 2. 1-g SAR (W/kg) with different sizes of rings at different distances from the cubical phantom.

plotted as a function of the external circumference of the ring and the distance $d$ from the ring to the cubic head. The results can be seen in Fig. 2.

Fig. 2 shows that the maximal 1-g SAR was found when a ring nearly 1 wavelength in circumference $(52 \mathrm{~mm}$ diameter) was used. Smaller and larger rings had a lesser effect. The 1-g SAR with no ring can be seen in the figure where the circumference is zero. The distance from the ring to the cubical phantom also affects the 1-g SAR, with rings close to the cubical phantom having little effect. Larger effects were found with the ring positioned 8 to $16 \mathrm{~mm}$ from the cubical phantom. The largest effect is with the $\lambda$ circumference ring, $12 \mathrm{~mm}$ from the head. This combination increased the 1-g SAR by 7.4 times from 0.50 to $3.70 \mathrm{~W} / \mathrm{kg}$. The figure also shows that rings that are slightly larger than a wavelength in circumference and positioned at least $12 \mathrm{~mm}$ away from the cubical phantom can decrease the 1-g SAR. It is well understood, that a source illuminating a resonant metallic loop creates currents on the loop. With a Z- polarized plane wave or a Z-directed dipole, the currents are not uniform around the ring and the sides of the ring (parallel to the source polarization) have the maximum currents, while the currents are at a minimum on the top and bottom sections of the ring (perpendicular to the source). These currents induce magnetic fields in a similar pattern around the ring. The electric fields around the ring are opposite to the magnetic fields (with the maximum values at the top and bottom of the ring).

Fig. 3 shows the results of subtracting the local SAR values without the ring from the local SAR values calculated with the $\lambda$ ring placed $12 \mathrm{~mm}$ from the cubical phantom. Recollect, that propagation is in the $X$ direction. Crudely referring geometries to a human head; the $Y Z$ plane simulates the plane at the front of the face and the $X Y$ plane is the horizontal plane extending to the back of the head, see inserts in Fig. 3. NB. Fig. 3 $=\mathrm{SAR}_{\text {ring }}$ minus $\mathrm{SAR}_{\text {no_ring. }}$.

Fig. 3(a) shows that the ring significantly increased the SAR at the center of the front surface of the phantom (near the ring) and that the presence of the ring decreased the SAR around the edges of the front face. Therefore, the ring focuses energy away from the edges of the front surface of cubical phantom and towards the section of the phantom closest to the ring. Fig. 3(b) 


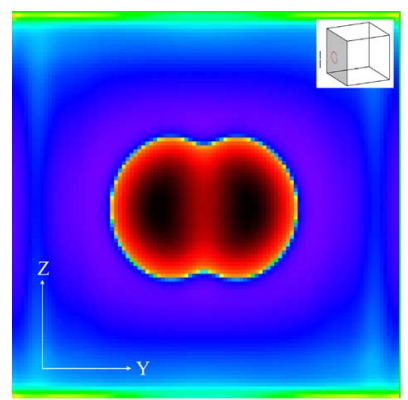

(a)

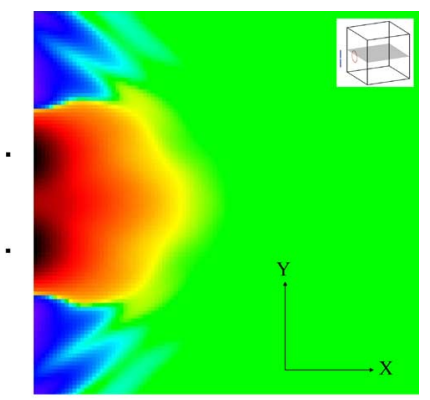

(b)

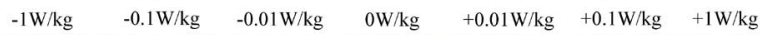

Fig. 3. The difference in Local SAR between the $\lambda$ ring and the no ring scenario in two cross sections (see inserts). (a) SAR difference at the surface of the liquid. (b) SAR difference on the central horizontal plane of the phantom. NB. the ring has been included in (b).
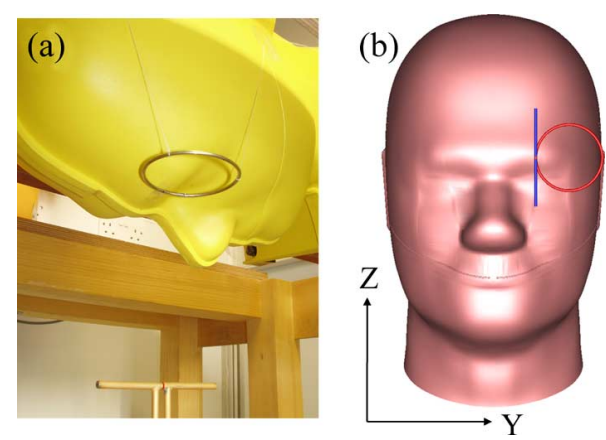

Fig. 4. A $\lambda$ ring near the eyebrow of the SAM head with a vertically orientated dipole. (a) Measurement setup. (b) SAM head in $Y Z$ plane.

shows the horizontal plane extending to the back of the head. As intuitively expected, the ring has little effect on the SAR in the back half of the head.

The area where the SAR is increased, at the front of the phantom, is not circular but approximately elliptical. with diameters of $66 \mathrm{~mm}$ in the $Y$ axis and $52 \mathrm{~mm}$ in the $Z$ axis. With the ring, there are two maximal regions of SAR (and increase in SAR as shown in Fig. 3). These occur in line with locations of the maximum current on the ring, as these high currents give rise to large magnetic fields, which are directly related to the SAR in the head [32].

\section{B. Measurements and Simulations With Phantom 2 at $1.8 \mathrm{GHz}$}

In these experiments, a modified Phantom head called the "Loughborough phantom" (modified rear-entry SAM head developed and tested by the authors) [30], [31] has been used. The head, which exists as Yee cells in an FDTD model and a fluid filled fiberglass human head in measurements, is based on the IEEE SAM head but has an opening at the rear to allow probing of the eyes cheeks and nose. The measurement setup is shown in Fig. 4 along with the geometry of the dipole and eyebrow ring added to Phantom 2. The figure shows that the ring touched the eyebrow and the rest of the ring was at different distances from the head. This makes the system more complex than the cubical phantom were the ring was always a constant distance from the head.

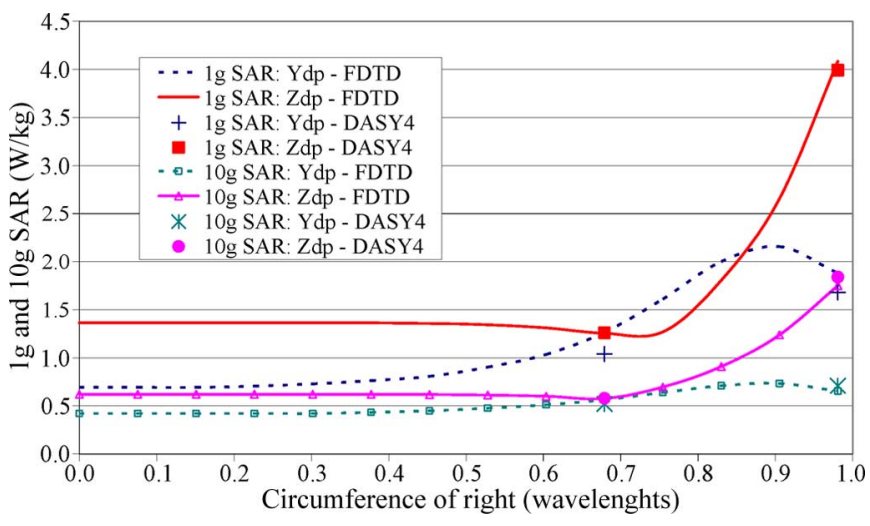

Fig. 5. DASY4 measurements and FDTD simulations with eyebrow ring near Phantom 2 with two orientations of dipole.

Both horizontally ( $Y$ axis) and vertically ( $Z$ axis) aligned dipoles are considered. Results were normalized to 1-W input power. The ring (made of $2 \mathrm{~mm}$ diameter copper wire), was attached to the eyebrow of the SAM phantom using cotton and clear sticky tape. Plumb lines and lasers were used to ensure that the alignment and position of both the ring and dipole were as accurate as possible. The measurements from the SAR robot system are made at $1.8 \mathrm{GHz}$, with two sizes of metallic ring.

The 1-g and 10-g SAR with the eyebrow ring are shown in Fig. 5. The 1-g SAR values are approximately double the 10-g SAR values. The DASY4 measurements show good agreement with the FDTD simulations for both the 1-g and 10-g SAR values. The largest SAR with the vertically orientated $Z$ dipole occurs with the ring which has a circumference of a wavelength. Whereas with the $Y$ orientated dipole, the ring resonates with a circumference of $\sim 0.9 \lambda$. With this orientation of dipole, the eyebrow causes the ring to be dielectrically loaded. Note, the dielectric loading phenomenon was not seen when the ring touched the head where the electric fields due to the ring are at a minimum, see Section III-A.

\section{Varying Ring Size and Position on Phantom 3 at $1.8 \mathrm{GHz}$}

Having shown the effect in homogenous phantoms by measurement and simulation, the results were extended to a more realistic head in FDTD.

In this section, jewelry rings have been added to the anatomically realistic Brooks head with a $Z$ orientated monopole on a box, positioned $80 \mathrm{~mm}$ in front of the nose, see Phantom 3 in Fig. 1. Rings of various sizes have been placed on the surface of the eyebrow, by the side of and below the nose. The rings near the nose were positioned level with the tip of the nose along the $X$ axis. The effect of piercing the rings through the skin has also been considered. This was done by moving the ring $4 \mathrm{~mm}$ in the $X$ direction away from the dipole. Part of the ring then lay inside the head and the copper Yee cells of the ring replaced the dielectric tissues of the head.

The results with the rings and the anatomical head are shown in Fig. 6. The results are similar to the rings with the SAM head in Fig. 2. The eyebrow ring and the ring by the side of the nose resonate when the circumference is approximately a wavelength. The ring below the nose resonates when the circumference is $\sim 0.7 \lambda$, as the nose causes dielectric loading in this orientation. However, the resonance size is less than with the ring near the eyebrow with the $Y$ aligned dipole, see Fig. 5 as there is 


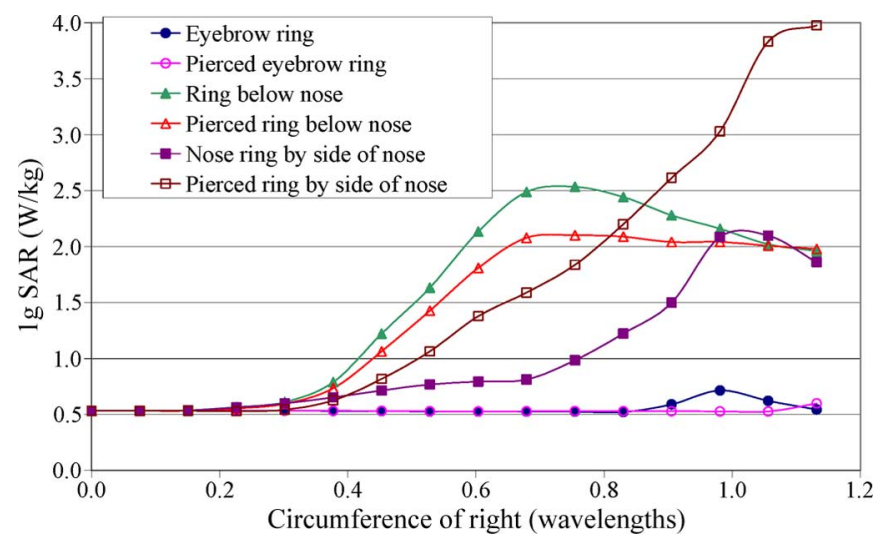

Fig. 6. The 1-g SAR with jewelry rings added to the Brooks head.
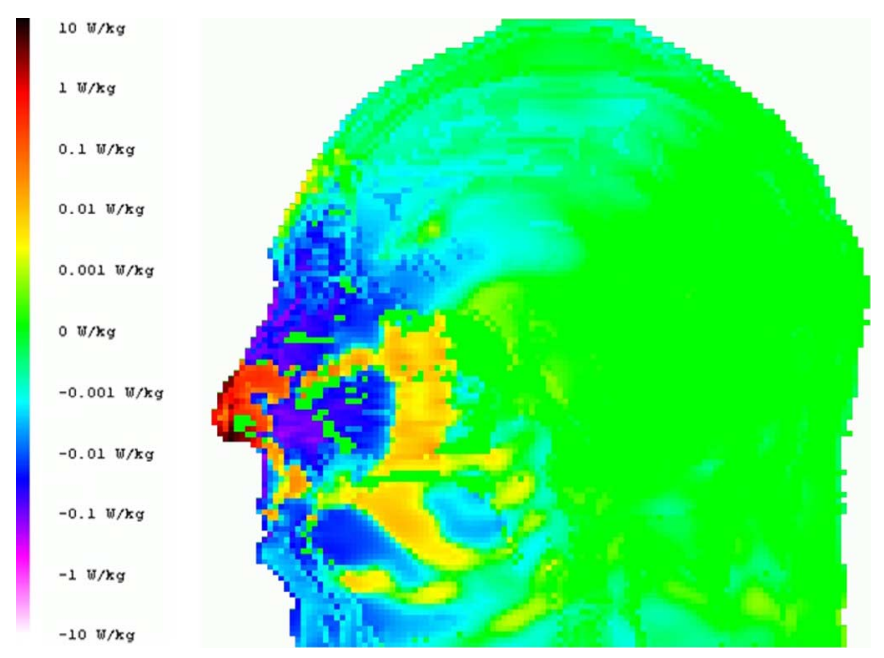

Fig. 7. The difference in SAR with the $60 \mathrm{~mm}$ diameter ring pierced by the side of the nose minus the SAR with no ring in a cut through the nose in the $\mathrm{XZ}$ plane. The ring increases the SAR in the nose and decreases the SAR above and below and behind the nose.

more contact between the ring and the head, see Fig. 4, and the tissue properties of the model are different. The eyebrow ring has little effect on the maximum 1-g SAR in the head when the monopole is placed in front of the tip of the nose. Note, that with the source in front of the nose, the maximum SAR occurs in the nose. Therefore, the $\lambda$ ring increased the SAR by the eyebrow, but had negligible effect on the maximum SAR in the head, as shown in Fig. 6.

Piercing the ring through the skin can both increase and decrease the 1-g SAR, compared to a ring touching the surface, depending on the location of the ring, as shown in Fig. 6. Piercing the ring through the skin by the eyebrow or by the side of the nose can increase the resonant size of the ring. The largest 1-g SAR occurs with a $1.13 \lambda$ ring pierced through the side of the nose of the Brooks head. This ring increased the 1-g SAR by 7.5 times to $3.98 \mathrm{~W} / \mathrm{kg}$. All the sizes and positions of jewelry investigated, increased the 1-g SAR in the anatomical head, compared to the case without the ring.

Fig. 7 presents results for the difference in SAR distribution caused by a $60 \mathrm{~mm}$ diameter ring. The change is most pronounced on the tip of the nose whilst at the back of the head there is little or no change in SAR.

\section{CONCLUSION AND DISCUSSION}

This paper has investigated and quantified the effects of circular metallic jewelry on the SAR in the head. The 1-g SAR was found to increase by approximately 7.5 times when circular metallic jewelry is placed inbetween the phantom and the RF source so that it is in-line with peak SAR location with the homogenous cubic phantom, the homogeneous realistically shaped phantom and the anatomically realistic head. The 1-g and 10-g SAR values obtained with a resonant ring were generally less than found in the literature with a mobile phone held to the ear.

Initially, a simple cubic phantom was used to explain the mechanisms that increased the SAR. Rings positioned away from the head were found to resonate when the circumference was approximately one wavelength. Rings entirely laying on the surface of the head had little effect. In a cubic phantom, rings were found to focus energy away from the edges of the front surface of the phantom and towards the middle of the front surface of the phantom.

Similar effects were found with rings touching the SAM head. The SAR was increased if the circumference of the ring is approximately a wavelength. The simulation results were verified with measurements using the DASY4 measurement system. The asymmetric current on the ring meant the ring behaved differently depending on where it was positioned on the head and the polarization of the source. Rings that touched the head, where the electric fields from the ring were large, resonated at a smaller size due to the head causing the ring to be dielectrically loaded. This would mean that smaller (and possibly more popular) jewelry rings may have an effect in certain geometries.

The understanding of the basic mechanisms found with simple phantoms was then extended to realistic jewelry, placed on an anatomical head with a realistic source. As before, the 1-g SAR increased when the ring was approximately a wavelength in circumference. The location of the ring relative to the skin and the head was found to be significant. Piercing the ring through the skin affected the SAR. Future work will consider jewelry in different positions, relative to the body, and different frequencies of excitation. Different anatomical phantoms will also be considered, as the shape and internal structure of each head is different and jewelry may have different effects on different people. A final point to note is that jewelry rings of the size needed to cause significant effects were large at $1.8 \mathrm{GHz}$ and although jewelry of this size is sometimes worn, it is not common.

\section{REFERENCES}

[1] A. D. Tinniswood, C. M. Furse, and O. P. Gandhi, "Computations of SAR distributions for two anatomically based models of the human head using CAD files of commercial telephones and the parallelized FDTD code," IEEE Trans. Antennas Propag., vol. 46, pp. 829-833, 1998.

[2] O. Gandhi, G. Lazzi, and C. M. Furse, "Electromagnetic absorption in the human head and neck for mobile telephones at 835 and $1900 \mathrm{MHz}$," IEEE Trans. Microw. Theory Technol., vol. 44, pp. 1884-1897, 1996.

[3] P. J. Dimbylow and S. M. Mann, "SAR calculations in an anatomically realistic model of the head for mobile communication transceivers at 900-MHz and 1.8-GHz," Phys. Med. Biol., vol. 39, pp. 1537-1553, 1994.

[4] P. Wainwright, "Thermal effects of radiation from cellular telephones," Phys. Med. Biol., vol. 45, pp. 2363-2372, 2000.

[5] L. Martens, J. Demoerloose, D. Dezutter, J. Depoorter, and C. Dewagter, "Calculation of the electromagnetic-fields induced in the head of an operator of a cordless telephone," Radio Sci., vol. 30, pp. 283-290, 1995. 
[6] W. Whittow, C. J. Panagamuwa, R. Edwards, J. C. Vardaxoglou, and P. McEvoy, "A study of head worn jewelry, mobile phone RF energy and the effect of differing tissue types on rates of absorption," presented at the 1st Eur. Conf. Antennas Propag. (EuCAP 2006), Nice, France, 2006.

[7] W. G. Whittow, C. J. Panagamuwa, R. M. Edwards, and J. C. Vardaxoglou, "The SAR effects of popular jewellery on the human head," presented at the 2nd Eur. Conf. Antennas Propag. (EuCAP 2007), Edinburgh, U.K., 2007.

[8] H. Virtanen, J. Keshvari, and R. Lappalainen, "The effect of authentic metallic implants on the SAR distributions of the head exposed to 900 , 1800 and $2450 \mathrm{MHz}$ dipole near field," Phys. Med. Biol., vol. 52, pp. 1221-1226, 2007

[9] W. Whittow, C. Panagamuwa, R. Edwards, and J. Vardaxoglou, "Specific absorption rates in the human head due to circular metallic earrings at $1800 \mathrm{MHz}$," in Proc. Loughborough Antennas Propag. Conf., Loughborough, U.K., 2007, pp. 277-280.

[10] J. Fayos-Fernandes, C. Arranz-Faz, A. Martinez-Gonzalez, and D. Sanchez-Hernandez, "Effect of pierced metallic objects on SAR distributions at 900 MHz," Bioelectromagn., vol. 27, pp. 337-353, 2006.

[11] J. Cooper and V. Hombach, "Increase in specific absorption rate in humans from implantations," Electron. Lett., vol. 32, pp. 2217-2219, 1996.

[12] H. Virtanen, J. Huttunen, A. Toropainen, and R. Lappalainen, "Interaction of mobile phones with superficial passive implants," Phys. Med. Biol., vol. 50, pp. 2689-2700, 2005.

[13] H. Virtanen, J. Keshvari, and R. Lappalainen, "Interaction of radio frequency electromagnetic fields and passive metallic implants-a brief review," Bioelectromagn., vol. 27, pp. 431-439, 2006.

[14] R. Tay, Q. Balzano, and N. Kuster, "Dipole configurations with strongly improved radiation efficiency for hand-held transceivers," IEEE Trans. Antennas Propag., vol. 46, pp. 798-806, 1998.

[15] J. Cooper and V. Hombach, "The specific absorption rate in a spherical head model from a dipole with metallic walls nearby," IEEE Trans. Electromagn. Compat., vol. 40, pp. 377-382, 1998.

[16] P. Bernardi, M. Cavagnaro, and S. Pisa, "Evaluation of the SAR distribution in the human head for cellular phones used in a partially closed environment," IEEE Trans. Electromagn. Compat., vol. 38, pp. 357-366, 1996

[17] W. G. Whittow and R. M. Edwards, "A study of changes to specific absorption rates in the human eye close to perfectly conducting spectacles within the radio frequency range 1.5 to $3.0 \mathrm{GHz}$," IEEE Trans. Antennas Propag., vol. 52, pp. 3207-3212, 2004.

[18] W. G. Whittow and R. M. Edwards, "Applications of a genetic algorithm for identification of maxima in specific absorption rates in the human eye close to perfectly conducting spectacles," Proc. Inst. Elect. Eng. Sci., Meas. Technol., vol. 152, pp. 89-96, 2005.

[19] S. E. Troulis, W. G. Scanlon, and N. E. Evans, "Effect of 'hands-free' leads and spectacles on SAR for a $1.8 \mathrm{GHz}$ cellular handset," in Proc. 1st Joint IEI/IEE Symp. Telecommun. Syst. Res., Dublin, Ireland, 2001, pp. $1675-1684$.

[20] J. Wang, T. Joukou, and O. Fujiwara, "Localized specific absorption rate in the human head in metal-framed spectacles for $1.5 \mathrm{GHz}$ handheld mobile telephones," Trans. IEE Japan, vol. 118-A, pp. 1234-1240, 1998.

[21] E. Joo, A. Szasz, and P. Szendro, "Metal-framed spectacles and implants and specific absorption rate among adults and children using mobile phones at 900/1800/2100 MHz," Electromagn. Biol. Med., vol. 25, pp. 103-112, 2006.

[22] D. W. Griffin, "A microwave antenna method of measuring the effect of metal-framed spectacles on microwaves near the eye," in Proc. Antennas Propag. Soc. Int. Symp., 1983, vol. 21, pp. 253-256.

[23] V. Anderson and K. H. Joyner, "Specific absorption rate levels measured in a phantom head exposed to radiofrequency transmissions from analog hand-held mobile phones," Bioelectromagn., vol. 16, pp. 60-69, 1995.

[24] J. P. Berenger, "A perfectly matched layer for the absorption of electromagnetic waves," J. Computat. Phys., vol. 114, pp. 185-200, 1994.

[25] P. J. Dimbylow and O. P. Gandhi, "Finite-difference time-domain calculations of SAR in a realistic heterogeneous model of the head for plane-wave exposure from $600 \mathrm{MHz}$ to $3 \mathrm{GHz}$," Phys. Med. Biol., vol. 36, pp. 1075-1089, 1991.

[26] F. Schonborn, M. Burkhardt, and N. Kuster, "Differences in energy absorption between heads of adults and children in the near fields of sources," Health Phys., vol. 74, pp. 160-168, 1998

[27] J. Keshvari and S. Lang, "Comparison of radio frequency energy absorption in ear and eye region of children and adults at 900, 1800 and 2450 MHz," Phys. Med. Biol., vol. 50, pp. 4355-4369, 2005.
[28] A. Hirata and T. Shiozawa, "Correlation of maximum temperature increase and peak SAR in the human head due to handset antennas," IEEE Trans. Microw. Theory Tech., vol. 51, pp. 1834-1840, 2003.

[29] A. Lee, H. Choi, J. Choi, and J. Pack, "Specific absorption rate values of handsets in cheek position at $835 \mathrm{MHz}$ as a function of scaled specific anthropomorphic mannequin models," ETRI J., vol. 27, pp. 227-230, 2005.

[30] C. J. Panagamuwa, W. Whittow, R. Edwards, J. C. Vardaxoglou, and P. McEvoy, "A study of the validation of RF energy specific absorption rates for simulations of anatomically correct head FDTD simulations and truncated DASY4 standard equipment measurements," presented at the 1st Eur. Conf. Antennas Propag. (EuCAP 2006), Nice, France, 2006.

[31] C. J. Panagamuwa, W. G. Whittow, R. M. Edwards, and J. C. Vardaxoglou, "Experimental verification of a modified specific anthropomorphic mannequin (SAM) head used for SAR measurements," in Proc. Loughborough Antennas Propag. Conf., Loughborough, U.K., 2007, pp. 261-264.

[32] N. Kuster and Q. Balzano, "Energy absorption mechanism by biological bodies in near field of dipole antennas above $300 \mathrm{MHz}$," IEEE Trans. Veh. Technol., vol. 41, pp. 17-23, 1992.

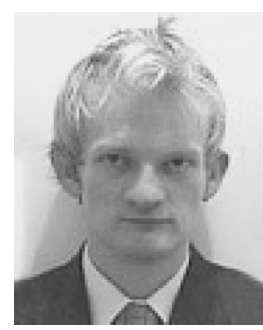

William G. Whittow received the B.Sc. degree in physics from The University of Sheffield, Sheffield, U.K., in 2000 and the Ph.D. degree from the EEE Department, Sheffield.

$\mathrm{He}$ is currently a Research Associate with Loughborough University, Leicestershire, U.K. His research interests include SAR, FDTD, bioectromagnetics, phantoms, and genetic algorithms.

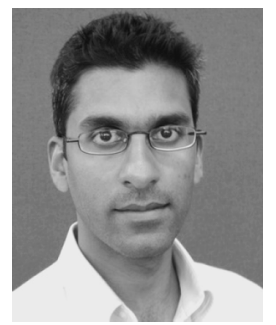

Chinthana J. Panagamuwa received the M.Eng. degree in electronic and electrical engineering in 2000, and the Ph.D. degree with a thesis on optically controlled microwave switches and frequency reconfigurable antennas in 2005, both from Loughborough University, Leicestershire, U.K.

$\mathrm{He}$ is currently a Lecturer in Photonic Systems at Loughborough University.

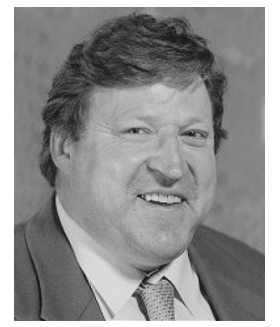

Robert M. Edwards (M'00-SM'08) read in electronic engineering with communications at the University of Sheffield, U.K., and studied for the Ph.D. degree with the Communications and Radar Group, University of Sheffield.

He moved to Loughborough University, Leicestershire, U.K., with his research group in September 2004. He was the Director of the University of Sheffield's Centre for Mobile Communications Research (C4MCR). He currently lectures Mobile Communications and is Head of the Communications Research Division, Loughborough University.

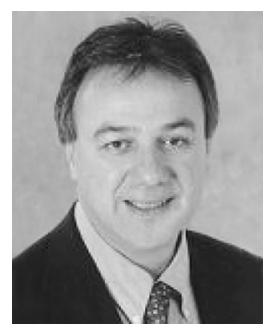

John (Yiannis) C. Vardaxoglou (M' 88) received the B.Sc. degree in mathematical physics in 1982 and the $\mathrm{Ph}$.D. degree in electronics in 1985 from the University of Kent, U.K

$\mathrm{He}$ joined Loughborough University, Leicestershire, U.K., as a lecturer in 1988. He was then promoted to Senior Lecturer in 1992 and Professor or Wireless Communications in 1998. He is currently Head of the Electronic and Electrical Engineering Department, Loughborough University. 